\section{Zeitschrift \\ für Wärmebehandlung und Werkstofftechnik}

vereinigt mit Härterei-Technik und Wärmebehandlung

\section{Herausgeber}

Im Auftrage der Arbeitsgemeinschaft Wärmebehandlung und Werkstofftechnik e. V. (AWT) herausgegeben von: Prof. Dr.-Ing. Johann Grosch Institut für Werkstofftechnik

Technische Universität Berlin Straße des 17. Juni 135, D-1000 Berlin 12

Dipl.-Ing. Rudi Jonck

Sonnenbühl 20, D-7000 Stuttgart 70

\section{Redaktion}

Prof. Dr.-Ing. Peter Mayr

Helga Bothe

Stiftung Institut für Werkstofftechnik

vorm. Institut für Härterei-Technik

Lesumer Heerstraße 32

D-2820 Bremen 77

Telefon (04 21) $630007 / 8$

Manuskripte sowie Mitteilungen, die den redaktionellen Inhalt betreffen, sind an die Schriftleitung zu senden.

\section{Organschaft}

Die Zeitschrift ist zugleich Organ der AWT - Arbeitsgemeinschaft Wärmebehandlung und Werkstofftechnik e.V Vorsitzender: Dr.-Ing. Hans Schlicht i. Hs. FAG Kugelfischer Georg Schäfer KGaA Postfach 1260, D-8720 Schweinfurt 2 Telefon: 097 21/913464

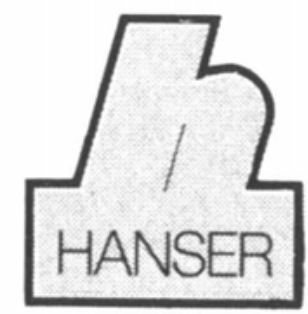

\section{Carl Hanser Verlag}

Kolbergerstraße 22, D-8000 München 80 Postanschrift:

Postfach 860420 , D-8000 München 86 Tel.-Sa.-Nr. (0 89) 9 26 94-0, Telex 5-22837 Fax: Infotec 089-984809

(C) Copyright by Carl Hanser Verlag 1986

\begin{tabular}{lr}
\hline Kurzfassungen & 110 \\
\hline Impressum & 112 \\
\hline Gefüge und Eigenschaften & \\
\hline $\begin{array}{l}\text { R. Chatterjee-Fischer, P. Mayr: Erzeugung und Untersuchung von } \\
\text { Mitteltemperatur-CVD-Hartstoffschichten ................... }\end{array}$ & 113 \\
$\begin{array}{l}\text { E. Rose, P. Mayr: Analyse von PVD/CVD-Verschleißschutzschichten } \\
\text { mit der Glimmentladungsspektroskopie (GDOS).............. }\end{array}$ & 127 \\
\hline Werkstoff- und Bauteilfestigkeit & \\
\hline
\end{tabular}

A. Korhonen, J. Pimiä, M. Sulonen: Einfluß einer nachfolgenden Wärmebehandlung auf die Haftung und die Eigenschaften von ionenplattierten TiN-Beschichtungen

\section{Technik der Wärmbehandlungsverfahren}

K. H. Kloos, E. Broszeit, T. Roth: Die Abscheidung von Hartstoffen nach den PVD-Verfahren.

H. R. Stock, P. Mayr: Hartstoffbeschichtung mit dem PlasmaCVD-Verfahren

D. Hofmann, J. Göbel, W.-D. Münz, M. Scherer, B. Hensel: Die Abscheidung von Nitrid- und Carbid-Hartstoffschichten zu Verschleißschutzzwecken und für andere Anwendungen mittels "Sputterlon-Plating"-Verfahren.

\section{Wärmebehandlungsanlagen und -mittel}

J. Vogel: Voraussetzungen und Einsatzmöglichkeiten für die Hartstoffbeschichtung von Präzisionswerkzeugen mittels PVD

\section{Sonderteil Aluminium}

T. Hirsch, O. Vöhringer, E. Macherauch: Der Einfluß des Kugelstrahlens auf die Biegeschwingfestigkeit von AlCu5Mg2 in verschiedenen Wärmebehandlungszuständen

\section{Produktberichte}

Präzisions-Mikro-Härteprüfer 159

Automatische Mikrohärtemessung nach dem Eindringverfahren.

Buchbesprechung

\title{
Zum Titelbild
}

Härten von homokinetischen Antriebsgelenken:

Vollautomatisches Härten der Kugellaufbahnen und des Schafts

\section{Durchsatz 400 Stück/Std.}

Energieversorgung $2 \times 150 \mathrm{~kW} 10 \mathrm{kHz}$ Speicherprogrammierbare Steuerung mit Fehlerdiagnosesystem und Qualitätssicherung über Prozessparameterkontrolle. Datenausgabe über Bildschirm und Drucker.

\section{HWG INDUCTOHEAT GMBH}

D-7313 Reichenbach/Fils

Ostweg 5, Postfach 1280

Telefon (0 7153) 5040

Telex 7266814

Fax $07153 / 504333$

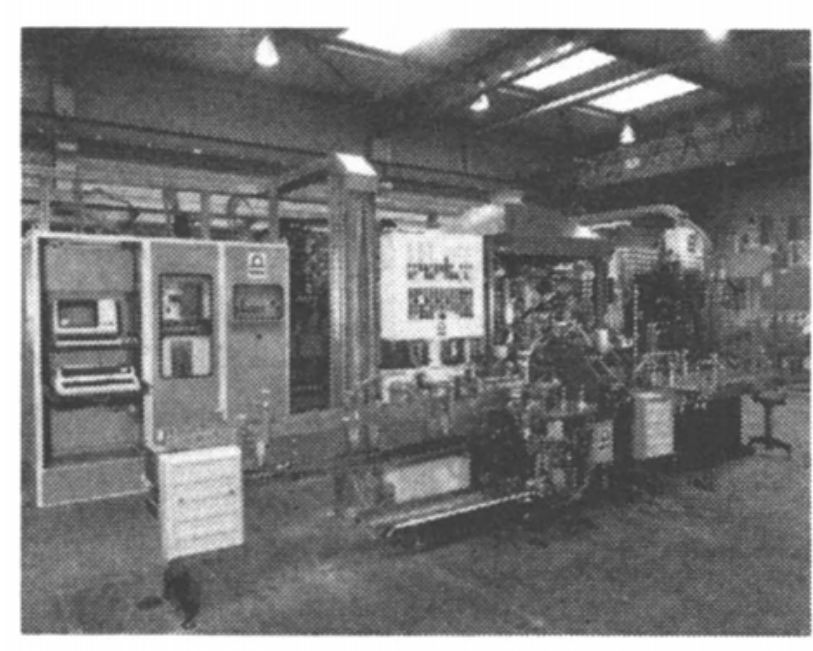

Präzision durch Induktion 